\title{
Gestión del Gobierno Abierto y uso del portal de transparencia en una universidad pública peruana
}

\section{(Management of Open Government and Use of the Transparency Portal in a Peruvian Public University)}

\author{
John Ruiz-Cueva1', Lloy Pinedo-Tuanama², Percy Barbarán-Mozo³ ,Luis Pretell-Paredes ${ }^{4}$
}

\begin{abstract}
Resumen
Las universidades peruanas están obligadas a articular sus políticas bajo un modelo de Gobierno Abierto (GA) según la Ley n²7 806 de Transparencia y Acceso a la Información Pública, con el propósito de garantizar la transparencia de la gestión institucional mediante la implementación de herramientas como portales web que garanticen una participación democrática digital. El objetivo del estudio fue determinar la relación entre la gestión del Gobierno Abierto y el uso del portal de transparencia en una universidad pública peruana. Para ello, se realizó una investigación básica de enfoque cuantitativo y diseño no experimental correlacional. Se aplicó una encuesta a través de dos cuestionarios a una muestra de 48 trabajadores encargados de publicar información. Los resultados y conclusiones revelan que el nivel de gestión del gobierno abierto y el uso de portal de transparencia son aceptables, con una correlación directa $(r=0.5)$ y con un sig. unilateral igual a 0.000 ( $p$ valor $<0.01)$. No obstante, existe desconfianza sobre la gestión del gobierno abierto y la utilidad del portal. Es decir, la institución superior adolece de estrategias o políticas de apertura gubernamental, lo que ocasiona baja participación pública en la gestión del gobierno universitario.
\end{abstract}

\section{Palabras clave}

Gobierno abierto, portal de transparencia, participación ciudadana, información pública.

\begin{abstract}
Peruvian universities are obliged to articulate their policies under an Open Government (GA) model according to Law $n^{\circ} 27806$ on Transparency and Access to Public Information, in order to guarantee the transparency of institutional management, through the implementation of tools, as web portals, that guarantee a democratic digital participation. The objective of the study was to determine the relationship between Open Government management and the use of the transparency portal in a Peruvian public university. To do this, a basic research with a quantitative approach and a correlational not experimental design was carried out. A survey was applied through two questionnaires to a sample of 48 workers in charge of publishing information. The results and conclusions reveal that the level of open government management and the use of a transparency portal are acceptable, with a direct correlation $(r=0.5)$ and with a sig. unilateral equal to 0.000 ( $p$ value $<0.01$ ). However, there is mistrust about the management of open government and the usefulness of the portal. In other words, the higher institution suffers from strategies or policies of government openness, which causes low public participation in the management of the university government.
\end{abstract}

\section{Keywords}

Open government, transparency portal, citizen participation, public information.

\footnotetext{
1 Universidad Nacional de San Martín. Tarapoto, Perú. [jaruiz@unsm.edu.pe, https://orcid.org/0000-0002-6820-8006] 2 Universidad Nacional de San Martín. Tarapoto, Perú. [lpinedo@unsm.edu.pe, https://orcid.org/0000-0002-5569-8739]

3 Universidad Nacional de San Martín. Tarapoto, Perú. [pbarbaran@unsm.edu.pe, https://orcid.org/0000-0002-9316-202X]

4 Agencia de Promoción de la Inversión Privada (Proinversión). Lima, Perú. [lpretell@proinversion.gob.pe, https://orcid. org/0000-0002-4989-4346]
} 


\section{Introducción}

La creciente digitalización en el sector público ha experimentado transformaciones que han sido calificadas como la segunda generación del desarrollo del gobierno electrónico (Estermann, 2018) y con ello la iniciativa bidireccional del Gobierno Abierto (GA) que, según Wirtz et al. (2018) mejora la transparencia institucional a través de la apertura de datos, promoviendo y expandiendo mecanismos de comunicación con el uso de las tecnologías de la información. Este modelo de gobierno es promovido por la Asociación de Gobierno Abierto (AGA) y requiere la participación conjunta de actores gubernamentales y sociedad civil para cumplir con el objetivo de modernización administrativa en un ambiente de democracia digital (Romero, 2019).

Según Castillo Sánchez et al. (2019), las universidades - consideradas instituciones del sector público o privado- están inmersas en el modelo de GA porque demandan del acceso a la información generada por procesos académicos y gestión administrativa. Un medio para incorporar el GA en las instituciones de educación superior es el uso de plataformas o portales web, basado en la participación ciudadana como principio fundamental (Moreno-Carmona et al., 2020) que permite ser transparente en la difusión de resultado científicos, reglamentos, procedimiento administrativos, entre otros (Saraite Sariene et al., 2020). Esta disposición de principios ha fomentado cambios para cumplir con los requisitos del desarrollo social académico responsable.

Para Porumbescu et al. (2020), el GA ha generado estudios de evaluaciones empíricas que demuestran que la realidad de su implementación es afectada por el desconocimiento del flujo de información y la baja comprensión de los ciudadanos, concibiendo la escasa oportunidad de participación. Así también lo manifiestan Wang y Shepherd (2020), quienes descubrieron que las medidas de apertura del GA son inadecuadas si la información es publicada en sitios web a modo informativo en lugar de datos abiertos, perjudicando los principios de transparencia.

En este contexto, Shepherd et al. (2019) evidencian limitantes que afectan la gestión del GA en sectores públicos de Inglaterra en donde se encuentran las instituciones de educación superior. Reflejan la incoherencia en la asignación de responsabilidades para la gestión de la política de datos abiertos, la falta de estándares de metadatos, diferencias en el personal sobre qué datos cargar en los portales, problemas de redundancia, inconsistencia e integridad, entre otros. Según Zuiderwijk y de Reuver (2021), estas dificultades se generan por las barreras críticas de funcionalidad y soporte, calidad y recursos de los datos, legislación, acceso y sostenibilidad.

Frente a ello, en Panamá, Castillo Sánchez et al. (2019) resaltan la pertinencia de asegurar la capacidad de los gestores del GA en estrategias de datos abiertos, considerando que las universidades (como fuentes de información y conocimiento) deben democratizar los datos y robustecer una cultura académica y gubernamental transparente. De acuerdo con Plantinga y Adams (2021), la implementación de GA debe integrar datos de la gestión administrativa, involucrando proactivamente a sus usuarios para así fomentar una participación satisfactoria, cumpliendo las expectativas de obtención de información.

Perú, por su parte, se integra a la AGA desde el 2012 y bajo lineamientos cuenta con una Estrategia Nacional de Datos Abiertos Gubernamentales y un Modelo de Datos Abierto Gubernamentales (Maldonado-Lozano et al., 2020) que compromete al modelo de GA a constituirse en un eje transversal de transparencia y acceso a la información en el marco de la Política Nacional de Modernización de la Gestión Pública articulado a la vigente Ley n² 27806 de Transparencia y Acceso a la Información Pública (Congreso de la República, 2003); así como al Decreto Supremo n 063-2010 que aprueba la implementación de portales de transparencia estándar en la administración pública (Presidencia del Consejo de Ministros, 2010). 
Sin embargo, pese a la obligatoriedad y esfuerzos desarrollados -rescatando, por supuesto, el crecimiento de la inclusión del GA por las instituciones públicas peruanas (Huamán Huamaní, 2020) - los resultados aún muestran deficiencias, ya que a partir del reporte de Viz-

carra Mamai et al. (2020) sobre GA se infiere que las entidades públicas incumplen con la divulgación oportuna y completa de la información en sus portales de transparencia, básicamente por la falta de capacidad de los encargados en gestionarlos.

Ahora bien, el presente estudio gira en torno a la problemática que se ha identificado en la Universidad Nacional de San Martín (UNSM), la cual está obligada a publicar información por la Ley de Transparencia y Acceso a la Información Pública Art.10 ( $n^{\circ} 27$ 806) y la Ley Universitaria Art.11 ( $n^{\circ} 30$ 220). Se reconoce que desde 2016 dicha institución ha implementado normativas y procedimientos para la actualización de su portal institucional y portal de transparencia. No obstante, la realidad evidencia la publicación parcial de información sobre contrataciones de bienes y servicios, planeamiento, organización y datos generales; así como la inexistencia de información sobre actividades oficiales, registros de visitas a funcionarios, entre otros.

Se propone como objetivo determinar la relación de la gestión del GA con el uso del portal de transparencia en la UNSM a partir de la identificación del nivel de cumplimiento de las mismas y el establecimiento de relaciones entre las dimensiones gestión administrativa, ingeniería del soporte informático, accesibilidad, relevancia y confiabilidad.

Bajo este paradigma se planteó la hipótesis general: existe relación directa y significativa entre la gestión del GA y el uso del portal de transparencia en la UNSM. Cabe resaltar la escasa literatura sobre el contexto del GA relacionado a portales de transparencia en universidades dentro del ámbito nacional e internacional. Pues, en su mayoría, derivan de estudios relacionados a ministerios y gobiernos locales. Por ende, la importancia del presente artículo en el aporte a la comunidad académica y la generación de posibles estudios sobre la misma línea de investigación.

\section{Metodología}

Se empleó un estudio básico de enfoque cuantitativo y diseño no experimental correlacional de corte transversal, ya que se buscó determinar la relación entre las variables: gestión de GA y uso del portal de transparencia en la UNSM en un periodo determinado. Para Arias (2006), este tipo de investigación aporta indicios sobre posibles causas de un fenómeno y su utilidad radica en predecir el valor aproximado de una variable sobre un determinado grupo a partir de la valoración de variables relacionadas.

La población estuvo conformada por 56 trabajadores docentes y administrativos de la UNSM encargados de publicar información en el portal web de transparencia durante el año 2017. Para la selección de la muestra se incluyó a todos los trabajadores designados mediante resolución universitaria y se excluyó a aquellos que, en el momento del estudio y a pesar de la designación mediante acto resolutivo, estuvieron laborando en otras áreas u oficinas. Es decir, se aplicó un tipo de muestreo no probabilístico intencional por accesibilidad, obteniendo una muestra de 48 trabajadores.

Las técnicas de recolección de datos fueron encuesta y uso dos cuestionarios; uno para medir el nivel de gestión del GA que constó de 14 ítems distribuidos en las dimensiones de gestión administrativa e ingeniería del soporte informático; el otro cuestionario sobre el uso del portal de transparencia que constó de 20 ítems dimensionados en accesibilidad, relevancia y confiabilidad. Ambos instrumentos tuvieron como opción de respuesta una escala valorativa 
tipo Likert. Además, fueron validados a través del juicio de expertos con una confiabilidad de 0.91 según Alpha de Cronbach.

Los datos recolectados fueron ordenados y procesados con Microsoft Excel y el SPSS versión 22. A partir de ello, se logró aplicar la técnica de estadística descriptiva para el análisis de la frecuencia absoluta, media y desviación estándar. A su vez, según la prueba de normalidad de Shapiro Wilk, se aplicó el coeficiente de correlación de Pearson.

Cabe señalar que la información institucional de la UNSM es de acceso público y no existe restricción que sitúe en riesgo a la institución, salvo lo descrito en la Ley n 27806 de transparencia y acceso a la información pública en Perú. No obstante, se solicitaron permisos para que los trabajadores seleccionados como unidad muestral de las diferentes oficinas administrativas y académicas brinden las facilidades para la ejecución del estudio.

\section{Resultados y discusión}

La tabla 1 muestra que los datos recopilados y procesados presentan alta dispersión y heterogeneidad según la desviación estándar en las puntuaciones de cada variable. La gestión del GA tuvo una media de 30 puntos y el uso del portal de transparencia es 43.06 puntos, lo que define que la percepción de los docentes y personal administrativo encargados de publicar información en el portal de transparencia de la UNSM fueron diversas.

Tabla 1. Medidas estadísticas sobre la gestión del GA y uso del portal de transparencia

\begin{tabular}{|c|c|c|c|c|c|c|}
\hline Variables & N & Rango & Mínimo & Máximo & Media & $\begin{array}{c}\text { Desviación } \\
\text { estándar }\end{array}$ \\
\hline Gestión del GA & 48 & 31 & 13 & 44 & 30 & \pm 6.776 \\
\hline Uso del portal de transparencia & 48 & 42 & 25 & 67 & 43.06 & \pm 9.894 \\
\hline
\end{tabular}

Según la tabla 2, en relación a la gestión del GA sobresalió el nivel 'aceptable' (61%) en cuanto a la gestión administrativa en el plan operativo institucional, estatutos y reglamentos, sistemas de control interno y la asignación de personal y disponibilidad presupuestal. Del mismo modo, para la ingeniería de soporte informático con tiempos definidos para la publicación de información, disponibilidad de equipos informáticos, equipo de trabajo, implementación de sistemas de seguridad, sistemas de respaldo de información y aplicación de los principios de la web 2.0. Sin embargo, se tuvo mínima proporción (4 \%) de consideración 'buena' y resaltó además una percepción de 'deficiente' (35\%).

Tabla 2. Percepción de la gestión de GA

\begin{tabular}{|c|c|c|c|}
\hline \multicolumn{2}{|c|}{ Nivel } & Cantidad & Porcentaje \\
\hline Bueno & $42-56$ & 2 & $4 \%$ \\
\hline Aceptable & $28-41$ & 29 & $61 \%$ \\
\hline Deficiente & $0-27$ & 17 & $35 \%$ \\
\hline \multicolumn{2}{r|}{ Total } & 48 & $100 \%$ \\
\hline
\end{tabular}

Estos resultados evidencian la baja internalización de gestionar políticas de GA por parte de los agentes encargados de velar por el acceso a información transparente, oportuna y con- 
fiable al conjunto de académicos, estudiantes y sociedad en general. Por su parte, en México, Vera Martínez (2018) manifestó que las medidas de gestión o planificación de políticas de GA en instituciones públicas aún se encuentran en desarrollo, exponiendo la necesidad de plantear marcos específicos que permitan tomar acciones estratégicas para la gestión eficiente de GA.

En cuanto al uso del portal de transparencia, en la tabla 3 se aprecia que el $54 \%$ del personal docente o administrativo señaló que su utilidad es 'aceptable' en cuanto a la accesibilidad de la información, ya que se publican documentos de planes y políticas institucionales, así como información académica, inversión y obras de infraestructura. Esta percepción fue igual para la relevancia y confiabilidad de la información, puesto que presenta estándares de atención definidos, interoperabilidad de datos, utilidad de información reusable y publicaciones en formatos reutilizables de calidad, consistentes y con la posibilidad de ser corroborada.

Sin embargo, el 40 \% de los encuestados consideró que el nivel de uso del portal de transparencia es 'deficiente' y solo una mínima parte (6 \%) considera que es 'bueno', deduciéndose que la institución universitaria adolece de robustez informática para la gestión del portal web. Según Zuiderwijk y de Reuver (2021), esto puede deberse a barreras críticas de funcionalidad y soporte o calidad y recursos de los datos, así como la propia legislación, acceso y sostenibilidad. Por lo que las instituciones deben fortalecer la gestión de tecnologías de información para el uso de portales, ya que son recursos pertinentes para la gestión transparente de GA.

Tabla 3. Percepción del uso del portal de transparencia

\begin{tabular}{|c|c|c|c|}
\hline \multicolumn{2}{|c|}{ Nivel } & Cantidad & Porcentaje \\
\hline Bueno & $60-80$ & 3 & $6 \%$ \\
\hline Aceptable & $40-59$ & 26 & $54 \%$ \\
\hline Deficiente & $0-39$ & 19 & $40 \%$ \\
\hline \multicolumn{2}{r|}{ Total } & 48 & $10 \%$ \\
\hline
\end{tabular}

\section{Relación entre la gestión del GA y uso del portal de transparencia}

Para responder la hipótesis planteada, primero se realizó la prueba de normalidad de los datos sobre las puntuaciones de las variables. En esta se obtuvo un nivel de significancia de 0.935 (gestión del GA) y 0.41 (uso del portal de transparencia). Al ser superiores a 0.05 se asume que los datos presentan una distribución normal según la prueba de Shapiro-Wilk (Tabla 4).

Tabla 4. Prueba de normalidad

\begin{tabular}{|c|c|c|c|}
\hline \multirow{2}{*}{ Variables } & \multicolumn{3}{|c|}{ Shapiro-Wilk } \\
\cline { 2 - 4 } & Estadístico & gl & Sig. \\
\hline Gestión del GA & 0.989 & 48 & 0.935 \\
\hline Uso del portal de transparencia & 0.976 & 48 & 0.421 \\
\hline
\end{tabular}

Posteriormente, se realizó la prueba de correlación de Pearson a nivel de variables y dimensiones (Tabla 5). El grado de correlación entre la gestión del GA y el uso del portal de transparencia en la UNSM arrojó un valor de r=0.5. Es decir, existe correlación moderada con 
dirección positiva con un sig. equivalente a 0.000 ( $p$ valor<0.01), aceptándose la hipótesis propuesta. Esto significa que las dimensiones de gestión administrativa e ingeniería del soporte informático inciden directamente en el uso del portal de transparencia en sus dimensiones de accesibilidad, relevancia y confiabilidad.

Tabla 5. Resumen de las correlaciones respecto a la gestión del GA y el uso del portal de transparencia

\begin{tabular}{|c|c|c|c|c|c|c|c|c|}
\hline \multicolumn{9}{|c|}{ Correlaciones } \\
\hline \multicolumn{2}{|c|}{ Variables y dimensiones } & \multirow{2}{*}{ 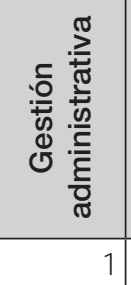 } & \multirow{2}{*}{ 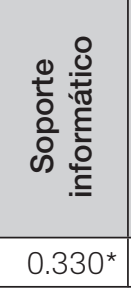 } & \multirow{2}{*}{ 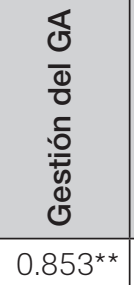 } & \multirow{2}{*}{ 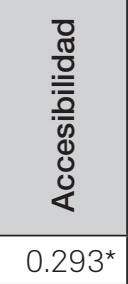 } & \multirow{2}{*}{\begin{tabular}{|l}
$\frac{\pi}{0}$ \\
$\frac{\pi}{\pi}$ \\
$\frac{\pi}{0}$ \\
$\frac{0}{0}$ \\
$\simeq$
\end{tabular}} & \multirow{2}{*}{ 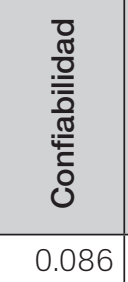 } & \multirow{2}{*}{ 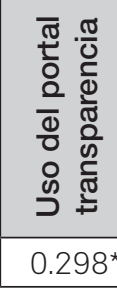 } \\
\hline & Correlación de Pearson & & & & & & & \\
\hline administrativa & Sig. (unilateral) & & 0.011 & 0.000 & 0.022 & 0.009 & 0.281 & 0.020 \\
\hline \multirow{2}{*}{$\begin{array}{c}\text { Soporte } \\
\text { informático }\end{array}$} & Correlación de Pearson & $0.330^{*}$ & 1 & $0.774^{\star \star}$ & $0.469^{* \star}$ & $0.533^{\star \star}$ & $0.427^{\star \star}$ & $0.543^{* *}$ \\
\hline & Sig. (unilateral) & 0.011 & & 0.000 & 0.000 & 0.000 & 0.001 & 0.000 \\
\hline \multirow{2}{*}{ Gestión del GA } & Correlación de Pearson & $0.853^{* *}$ & $0.774^{* *}$ & 1 & $0.455^{\star *}$ & $0.524^{\star \star}$ & $0.293^{*}$ & $0.500^{* *}$ \\
\hline & Sig. (unilateral) & 0.000 & 0.000 & & 0.001 & 0.000 & 0.022 & 0.000 \\
\hline \multirow{2}{*}{ Accesibilidad } & Correlación de Pearson & $0.293^{*}$ & $0.469^{* *}$ & $0.455^{\star *}$ & 1 & $0.586^{\star \star}$ & $0.694^{\star \star}$ & $0.936^{\star *}$ \\
\hline & Sig. (unilateral) & 0.022 & 0.000 & 0.001 & & 0.000 & 0.000 & 0.000 \\
\hline \multirow{2}{*}{ Relevancia } & Correlación de Pearson & $0.341^{* \star}$ & $0.533^{\star *}$ & $0.524^{\star *}$ & $0.586^{\star *}$ & 1 & $0.611^{* *}$ & $0.801^{* *}$ \\
\hline & Sig. (unilateral) & 0.009 & 0.000 & 0.000 & 0.000 & & 0.000 & 0.000 \\
\hline \multirow{2}{*}{ Confiabilidad } & Correlación de Pearson & 0.086 & $0.427^{* *}$ & $0.293^{*}$ & $0.694^{* *}$ & $0.611^{\star *}$ & 1 & $0.838 * *$ \\
\hline & Sig. (unilateral) & 0.281 & 0.001 & 0.022 & 0.000 & 0.000 & & 0.000 \\
\hline \multirow{3}{*}{$\begin{array}{l}\text { Uso del portal } \\
\text { transparencia }\end{array}$} & Correlación de Pearson & $0.298 *$ & $0.543^{\star *}$ & $0.500^{* *}$ & $0.936^{\star \star}$ & $0.801^{\star \star}$ & $0.838^{* \star}$ & 1 \\
\hline & Sig. (unilateral) & 0.020 & 0.000 & 0.000 & 0.000 & 0.000 & 0.000 & \\
\hline & $\mathrm{N}$ & 48 & 48 & 48 & 48 & 48 & 48 & 48 \\
\hline \multicolumn{9}{|c|}{ *La correlación es significativa en el nivel 0.05 (1 cola). } \\
\hline \multicolumn{9}{|c|}{ **La correlación es significativa en el nivel 0.01 (1 cola). } \\
\hline
\end{tabular}

Figura 1. Dependencia entre la gestión del GA y uso del portal de transparencia

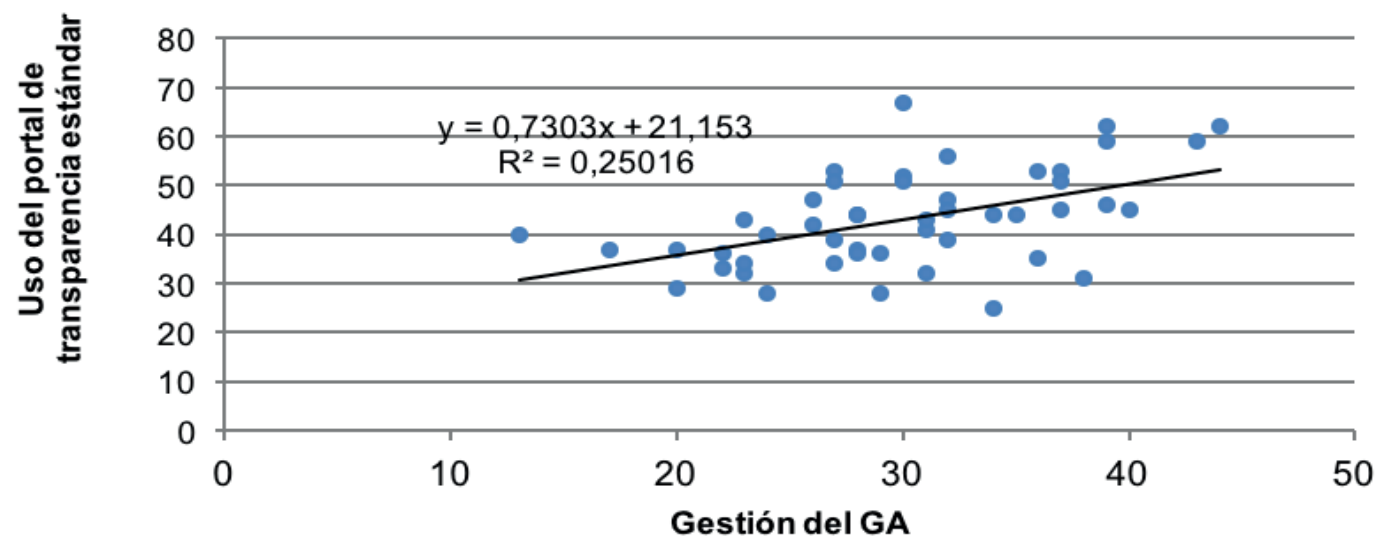


La figura 1 muestra que el uso del portal de transparencia en la institución universitaria depende de la gestión del GA en un $25 \%$ y la diferencia (75 \%) recae sobre otros factores ajenos a dicha gestión como: el cumplimiento del código de ética, gestión por resultados, cumplimiento de planes operativos anuales, inadecuada selección de personal y alta rotación de personal. Siendo el pilar del GA la transparencia de la información y que el uso del portal es la herramienta para transparentar la información pública, se presenta una baja dependencia. La razón de esta realidad se explica en que la institución ha descuidado el proceso de publicación de información debido a la rotación continua del personal administrativo y autoridades asignados a la gestión del GA, los mismos que desconocen el proceso. Este resultado coincide con lo expuesto por Shepherd et al. (2019), quienes manifestaron que la asignación de responsabilidad para la gestión de políticas de datos abiertos en entidades públicas sin evaluar las capacidades del recurso humano es una irregularidad que persiste en la dificultad del personal para gestionar los portales de transparencia, ocasionando problemas de redundancia e inconsistencia en la información expuesta.

De los resultados presentados en la Tabla 5 se destaca la correlación baja $(r=0.29)$ entre la confiabilidad y la gestión del GA con un sig. igual a 0.022 ( $p$ valor<0.05). Ello implica que el personal docente, administrativo o sociedad académica y civil desconfían de la gestión institucional bajo el modelo de GA, consecuentemente de la información publicada en el portal de transparencia. Esto genera una participación democrática limitada y afecta los principios de transparencia (Porumbescu et al, 2020).

Por su parte, Vizcarra Mamai et al. (2020) demostraron la existencia de correlación significativa directamente proporcional y positiva $(r=0.82)$ entre el GA y la participación ciudadana en una municipalidad peruana. Resultado semejante desde una perspectiva de confiabilidad de la gestión del GA en una institución universitaria. Esto puede deberse a la relación significativa $(r=0.69)$ entre el GA y la política gestionada por la municipalidad que guarda relación ( $r=0.85$ con un sig. igual a 0000 ( $p$ valor<0,01) entre la gestión administrativa y la gestión del GA.

Bajo esta perspectiva, en Argentina, Cazzolli et al. (2020) sostienen que las instituciones que solo brindan disponibilidad y accesibilidad de información pública en los portales de transparencia sin asegurar la participación del ciudadano en la gestión de gobierno reducen la potencialidad democratizadora del GA. Por consiguiente, Negrete-Huelga y Rivera-Magos (2018) manifiestan que la comunicación es tarea primordial de la apertura gubernamental para que exista colaboración y participación equitativa entre los agentes que gestionan el GA y la sociedad en general.

\section{Conclusiones y recomendaciones}

El nivel de percepción sobre la gestión del GA y el uso del portal de transparencia en la Universidad Nacional de San Martín es aceptable, con un nivel de correlación significativa y moderada $(r=0.5$ con un p valor igual a $0.000<0.01$ ). Sin embargo, existe un $25 \%$ de dependencia entre las variables. Esto refleja la baja gesta de estrategias o políticas institucionales para la apertura gubernamental de información pública. Hecho que trasciende la desconfianza del público sobre la gestión del GA y, consecuentemente, la baja participación digital de la sociedad académica y civil.

A partir del estudio, se fundamenta que la iniciativa de GA en una institución superior universitaria debe velar por el principio de transparencia institucional con la accesibilidad, relevancia y confiabilidad de los datos e información publicada en los portales de transparencia 
estándar. Para ello, es pertinente fortalecer las capacidades de los agentes encargados de la gestión del GA y garantizar la participación de los ciudadanos mediante estrategias de comunicación que los involucren en la gestión del gobierno democrático.

En ese sentido, esta investigación se constituye como un referente para futuros trabajos que deben centrarse en analizar la gestión del GA en instituciones universitarias desde una percepción más amplia que involucre tanto a funcionarios, docentes, estudiantes y personal administrativo, así como a la sociedad civil. Además, teniendo en cuenta los resultados desfavorables que califican como deficiente gestión del GA y uso del portal de transparencia estándar, se recomienda la realización de estudios aplicados en los cuales se diseñen estrategias o políticas institucionales para la gestión eficiente del GA y así asegurar la participación ciudadana, pilar de la transparencia en la práctica de GA.

\section{Referencias}

Arias, F. (2006). El proyecto de investigación. Introducción a la metodología científica. Editorial Episteme. Castillo, G.; Murillo, D.; Berbey, A., y Álvarez, H. (2019). El acceso a la información: una propuesta en Panamá de Gobierno Abierto enfocados a contenido académico. Memorias De Congresos UTP: 113120. https://revistas.utp.ac.pa/index.php/memoutp/article/view/2301

Cazzolli, A.; Echavarría, C., y Quevedo, C. (2020). Estrategias comunicacionales a partir de dispositivos de Gobierno Abierto en la provincia de Córdoba (Argentina). Revista de Tecnología y Sociedad, 10(19): 1-26. https://doi.org/10.32870/Pk.a10n19.549

Congreso de la República. (2003). Ley n² 27806.- Ley de Transparencia y Acceso a la Información Pública. Presidencia de Consejo de Ministros. https://www.peru.gob.pe/normas/docs/ley_27806.pdf

Estermann, B. (2018). Development Paths Towards Open Government. An Empirical Analysis Among Heritage Institutions. Government Information Quarterly, 35(4): 599-612. https://doi.org/10.1016/j. giq.2018.10.005

Huamán Huamaní, E. (2020). El Gobierno Abierto en el Perú: compromisos y una buena práctica de integridad electoral. Huamán Huamaní. Saber Servir: Revista de la Escuela Nacional De Administración Pública, 4: 146-170. http://revista.enap.edu.pe/article/view/4167

Maldonado-Lozano, A; Paredes-Aguilar, L., y Palomino Alvarado, G. (2020). Gestión de Gobierno Abierto en las instituciones públicas: una revisión de la literatura. Sapienza: International Journal of Interdisciplinary Studies, 2(2): 137-152. https://journals.sapienzaeditorial.com/index.php/SIJIS/ article/view/82

Moreno-Carmona, C.; Feria-Domínguez, J., y Troncoso, A. (2020). Applying the Open Government Principles to the University's Strategic Planning: A Sound Practice. Sustainability, 12(5): 1826. https://doi. org/10.3390/su12051826

Negrete-Huelga, K., y Rivera-Magos, S. (2018). Estrategias de comunicación en redes sociodigitales desde la práctica del Gobierno Abierto. Cuadernos. Info, 42: 183-196. https://doi.org/10.7764/ cdi. 42.1242

Plantinga, P., y Adams, R. (2021). Rethinking Open Government as Innovation for Inclusive Development: Open Access, Data and ICT in South Africa. African Journal of Science, Technology, Innovation and Development, 13(3): 315-323. https://doi.org/10.1080/20421338.2020.1746046

Porumbescu, G., Cucciniello, M., Y Gil-Garcia, J. (2020). Accounting for Citizens When Explaining Open Government Effectiveness. Government Information Quarterly, 37(2): 101451. https://doi. org/10.1016/j.giq.2019.101451

Presidencia del Consejo de Ministros. (2010). Decreto Supremo que aprueba la implementación del Portal de Transparencia Estándar en las Entidades de la Administración Pública. PMC. http://www. oas.org/juridico/PDFs/mesicic4_per_ds063.pdf 
Romero, L. (2019). Una aproximación teórica al Gobierno Abierto. Cuestiones Políticas, 34(60): 69-91. https://produccioncientificaluz.org/index.php/cuestiones/article/view/29127

Saraite, L.; Caba, C., y López, A. (2020). Expanding the Actions of Open Government in Higher Education Sector: From Web transparency to Open Science. PLOS ONE, 15(9): 1-18. https://doi.org/10.1371/ journal.pone.0238801

Shepherd, E., et al. (2019). Open Government Data: Critical Information Management Perspectives. Records Management Journal, 29(1/2): 152-167. https://doi.org/10.1108/RMJ-08-2018-0023

Vera Martínez, M. (2018). Los datos abiertos y el plan de acción de gobierno abierto en México 2013-2015. Nóesis. Revista de Ciencias Sociales y Humanidades, 27(54): 1-18. https://doi.org/10.20983/ noesis.2018.2.1

Vizcarra Mamai, F., et al. (2020). Gobierno electrónico y participación ciudadana en la municipalidad de Los Olivos 2018. Revista Inclusiones, 7: 160-180. http://www.revistainclusiones.org/index.php/ inclu/article/view/1203

Wang, V., y Shepherd, D. (2020). Exploring the Extent of Openness of Open Government Data. A Critique of Open Government Datasets in the UK. Government Information Quarterly, 37(1): 101405. https://doi.org/10.1016/j.giq.2019.101405

Wirtz, B.; Weyerer, J., y Rösch, M. (2018). Citizen and Open Government: An Empirical Analysis of Antecedents of Open Government Data. International Journal of Public Administration, 41(4): 308-320. https://doi.org/10.1080/01900692.2016.1263659

Zuiderwijk, A., y de Reuver, M. (2021). Why Open Government Data Initiatives Fail to Achieve Their Objectives: Categorizing and Prioritizing Barriers Through a Global Survey. Transforming Government: People, Process and Policy. https://doi.org/10.1108/TG-09-2020-0271 